\title{
Magnetic Tunnel Junction Based on MgO Barrier Prepared by Natural Oxidation and Direct Sputtering Deposition
}

\author{
Xiaohong Chen ${ }^{1,2, *}$, Paulo. P. Freitas ${ }^{2}$
}

(Received 12 December 2011; accepted 15 February 2012; published online 28 February 2012.)

\begin{abstract}
Magnetic tunnel junctions (MTJs) based on $\mathrm{MgO}$ barrier have been fabricated by sputtering single crystal $\mathrm{MgO}$ target and metal $\mathrm{Mg}$ target, respectively, using magnetic sputtering system Nordiko 2000. MgO barriers have been formed by a multi-step deposition and natural oxidization of $\mathrm{Mg}$ layer. $\mathrm{Mg}$ layer thickness, oxygen flow rate and oxidization time were adjusted and the tunnel magnetoresistance (TMR) ratio of optimal MTJs is over $60 \%$ at annealing temperature $385^{\circ} \mathrm{C}$. The $(001) \mathrm{MgO}$ crystal structure was obtained when the separation distance between $\mathrm{MgO}$ target and substrate is less than $6 \mathrm{~cm}$. The TMR ratio of most $\mathrm{MgO}$ based MTJs are over $100 \%$ at the separation distance of $5 \mathrm{~cm}$ and annealing temperature $340{ }^{\circ} \mathrm{C}$. The TMR ratios of MTJs are almost zero when the separation distance ranges from 6 to $10 \mathrm{~cm}$, due to the amorphous nature of the $\mathrm{MgO}$ film.
\end{abstract}

Keywords: Magnetic tunnel junctions; MgO; Crystal structure; Magnetic sputtering system

Citation: Xiaohong Chen and Paulo. P. Freitas, "Magnetic Tunnel Junction Based on MgO Barrier Prepared by Natural Oxidation and Direct Sputtering Deposition", Nano-Micro Lett. 4 (1), 25-29 (2012). http://dx. doi .org/10.3786/nml.v4i1.p25-29

\section{Introduction}

Since Julliere's research group investigated the first spin-dependent tunneling junctions based on Co and Fe as electrode materials and $\mathrm{GeO}$ as insulating barrier in 1974 [1], magnetic tunneling junctions (MTJs) have been extensively studied due to its promising applications in the hard disk read heads, magnetoresistive random access memory and sensors $[2,3]$. The MTJs based on different tunnel barriers such as $\mathrm{NiO}$ [4] and $\mathrm{Gd}_{2} \mathrm{O}_{3}$ [5], showed very low tunnel magnetoresistance (TMR) effects. The TMR breakthrough is that Miyarzaki et. al. group reported MTJs based on amorphous $\mathrm{Al}_{2} \mathrm{O}_{3}$ barriers attained TMR of $18 \%$ at room temperature in 1995 [6]. At the present, the optimal MTJs based on aluminum oxide barrier can reach TMR ratio of $80 \%$ [3]. However, it is difficult for MTJs based on $\mathrm{Al}_{2} \mathrm{O}_{3}$ barrier to further improve TMR ratio. According to the first-principle electronic structure calculations, the ordered (001) oriented Fe/MgO/Fe MTJs structure were suggested to reach TMR ratio more than $100 \%$ to even $1000 \%$ [7]. This giant TMR ratio was thought to be caused by an interfacial spin-dependent electronic state with $\Delta_{1}$ symmetry at the Fermi energy. Along this theoretical calculation, S. S. P. Parkin group [8] and Shinji Yuasa group [9] reported giant TMR ratio up to $220 \%$ and $180 \%$ at room temperature in 2004 , respectively. The higher TMR ratio of $604 \%$ was further reported in $\mathrm{CoFeB} / \mathrm{MgO}(001) / \mathrm{CoFeB}$ MTJs using magnetic sputtering deposition [2]. To realize high TMR in MTJs, it is important to deposit (001) $\mathrm{MgO}$ crystallographic orientation. Wang et al reported that an improved crystallinity of the $\mathrm{MgO}$ (001) layer is a main reason for increasing TMR ratio at high annealing temperature [10].

\footnotetext{
${ }^{1}$ Engineering Research Center for Nanophotonics and Advanced Instrument Ministry of Education, Department of Physics, East China Normal University, Shanghai 200062, China

${ }^{2}$ INESC-MN, R.Alves Redol 9, 100029 Lisboa, Portugal

*Corresponding author. E-mail: xhchen@phy.ecnu.edu.cn
} 
The excellent crystalline (001) MgO layer and (200) $\mathrm{CoFeB}$ layer are both primary factors for the higher $\mathrm{TMR}$ in $\mathrm{CoFeB} / \mathrm{MgO} / \mathrm{CoFeB}$ structure, confirmed by $\mathrm{X}$-ray diffraction [11].

The (001) orientation $\mathrm{MgO}$ layer has been fabricated with different methods, such as MBE [9], magnetic sputtering deposition $[8,10]$ and ion beam deposition [12], etc. The deposition parameters, such as Ar pressure [13], sputtering power, deposition rate and the distance between target and substrate would usually affect the crystal structure of $\mathrm{MgO}$ layer in magnetic sputtering systems. Therefore, the careful research work that the influence of deposition parameters on the crystal structure of $\mathrm{MgO}$ layer and the TMR ratio of $\mathrm{MgO}$ based MTJs is important for quickly and successfully fabricating (001) $\mathrm{MgO}$ crystal structure and obtaining higher TMR ratio of $\mathrm{MgO}$ based MTJs. In this study, we investigated MTJs based on $\mathrm{MgO}$ barrier fabricated by magnetic sputtering single crystal $\mathrm{MgO}$ target and metal $\mathrm{Mg}$ target, respectively. As for $\mathrm{MgO}$ barrier deposited with single crystal $\mathrm{MgO}$ target, the distance between $\mathrm{MgO}$ target and substrate is sensitive to the crystal structure of $\mathrm{MgO}$ layer. For $\mathrm{MgO}$ barrier prepared by sputtering metal $\mathrm{Mg}$ target, the metal $\mathrm{Mg}$ layer with several nanometers was naturally oxidized to form $\mathrm{MgO}$ barrier. The sputtering parameters of oxygen flow rate and pressure, oxidization time and $\mathrm{Mg}$ layer thickness apparently influence TMR ratio and the products of resistance and area (RA).

\section{Experimental Procedures}

The thin films for the MTJs were deposited onto glass substrate using a magnetron sputtering system (Nordiko 2000), with a base pressure of $5 \times 10^{-8}$ Torr. A magnetic field of 20 Oe was applied to induce parallel easy axis in the bottom and top magnetic electrodes during deposition. The fundamental structure of the MTJ is Ta70/Ru50/Ta50/MnPt200/CoFe25/Ru8/CoFeB30/ $\mathrm{MgO}\left(\mathrm{t}_{\mathrm{MgO}}\right) / \mathrm{CoFeB} 30 / \mathrm{Ta} 70 / \mathrm{TiWN} 150$ (in $\AA$ ). The $t_{\mathrm{MgO}}$ represents the nominal thickness of $\mathrm{MgO}$ barriers. For sputtering metal $\mathrm{Mg}$ target, $\mathrm{MgO}$ barriers were formed by a several-step natural oxidization. First, the $\mathrm{Mg}$ layer was deposited on top of bottom magnetic electrode at deposition rate of $0.26 \AA / \mathrm{s}$ in the main chamber, and then oxidized by pure oxygen to form $\mathrm{MgO}$ layer in the load-lock chamber. To fully oxidize $\mathrm{Mg}$ layer and get right thickness of $\mathrm{MgO}$ barrier, the process of depositing $\mathrm{Mg}$ layer and natural oxidization need to be repeated several times in sequence. For sputtering single crystal $\mathrm{MgO}$ target, $\mathrm{MgO}$ barriers were deposited by RF sputtering and their nominal thickness was varied from $15 \AA$ to $30 \AA$. The microsize junctions were patterned by a self-aligned micro- fabrication process using direct-write laser-lithography, ion-beam milling and lifting off, defining junction areas down to $1 \times 1 \mathrm{um}^{2}$. Patterned samples were annealed with different temperature in vacuum pressure of $10^{-6}$ Torr for $1 \mathrm{~h}$, and then moved into a magnetic field of $1 \mathrm{~T}$ while cooling down. The TMR transfer curves were measured with a four-contact DC method. The crystalline structure of $\mathrm{MgO}$ films was characterized by X-ray diffractometer (XRD, Siemens D-5000, molybdenum source $\mathrm{K} \alpha=0.7107 \AA$ ) [12].

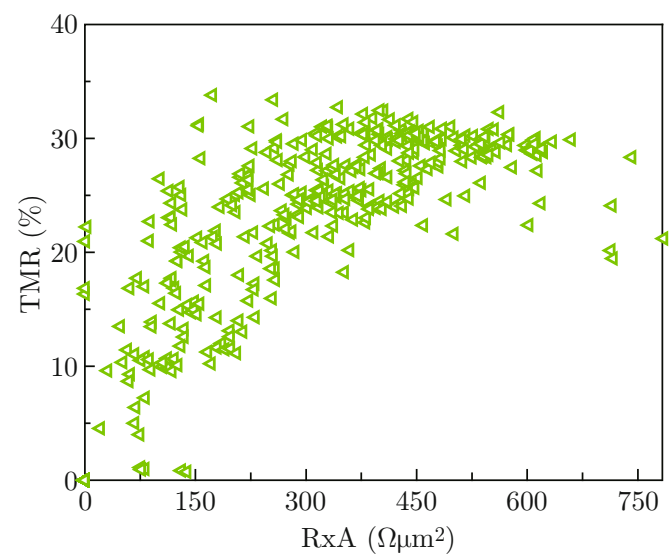

(a) $\mathrm{Mg}$ layer 3.8

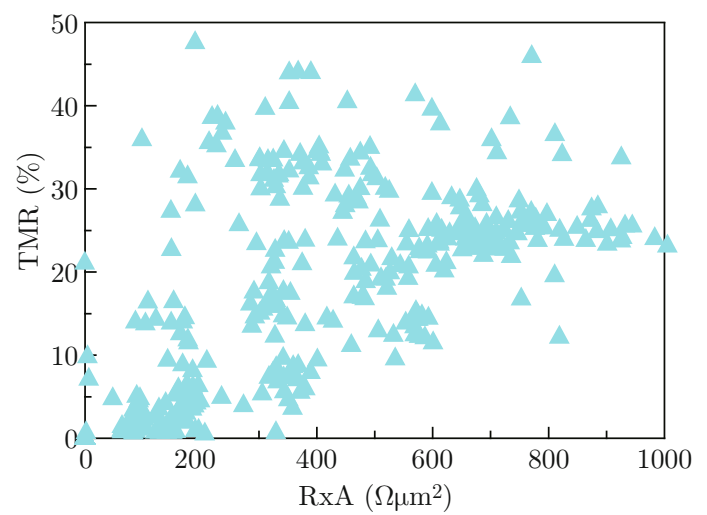

(b) Mg layer 6.24

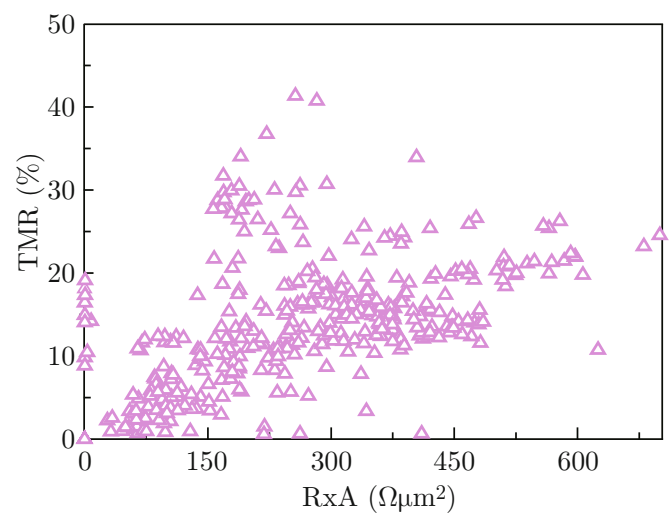

(c) $\mathrm{Mg}$ layer 7.6

Fig. 1 TMR ratios versus RA products for MTJs with different $\mathrm{Mg}$ layer thickness. 


\section{Results and Discussion}

Figure 1 shows TMR ratios versus RA products with different $\mathrm{Mg}$ layer thickness. The $\mathrm{Mg}$ layer thickness per step deposition was chosen $3.8 \AA, 6.24 \AA$ and 7.6 $\AA$, respectively. MTJs were annealed at $370^{\circ} \mathrm{C}$ for 1 hour. Data are from $\sim 324$ MTJs assemblage. The total nominal thickness of $\mathrm{Mg}$ layer was about $30 \AA$. The first $\mathrm{Mg}$ layer was oxidized for 40 seconds and the following several $\mathrm{Mg}$ layers were oxidized for 80 seconds at $2 \mathrm{sccm}$ oxygen flow rate. The shortened oxidization time of the first step is to avoid oxidizing the bottom electrode, which can decrease TMR of MTJs [14]. It seems difficult to judge which depositing $\mathrm{Mg}$ layer thickness is better for higher TMR ratio, as illustrated in Fig. 1. However, MTJs with $6.24 \AA \mathrm{Mg}$ layer are probably worthy further optimization because that the number of MTJs with $\mathrm{Mg}$ layer $6.24 \AA$ of more than $40 \%$ TMR is more than that of MTJs with $\mathrm{Mg}$ layer $7.6 \AA$ and $3.8 \AA$.

For MTJs with $\mathrm{Mg}$ layer $6.24 \AA$, TMR ratio versus RA products for different oxidization time was shown in Fig. 2. The oxygen flow rate of oxidizing $\mathrm{Mg}$ layer was $16 \mathrm{sccm}$. The MTJs were annealed at $370^{\circ} \mathrm{C}$ for $1 \mathrm{~h}$. The average RA products of MTJs with oxidization time 80 and 100 seconds are apparently larger than that of MTJs with 60 seconds due to the complete oxidization of these $\mathrm{Mg}$ layers [15]. However, the higher TMR ratios of MTJs with oxidization time 60,80 and 100 seconds are all over $40 \%$ at annealing temperature $370^{\circ} \mathrm{C}$. The TMR ratio of MTJs was further improved and obtained more than $60 \%$ at annealing temperature $385^{\circ} \mathrm{C}$, as shown in Fig. 2(d).

For $\mathrm{MgO}$ barriers deposited by single crystal $\mathrm{MgO}$ target, it is very important for high TMR ratio to obtain (001) $\mathrm{MgO}$ crystal structure [7,9]. Here, sputtering parameters, such as RF power, Ar gas flow, gas pressure and the separation distance between $\mathrm{MgO}$ target and glass substrate were optimized. However, our experiments indicated that the separation distance between $\mathrm{MgO}$ target and substrate is most important parameter in magnetic sputtering system Nordiko 2000. Fig. 3 shows the XRD scan curves of $\mathrm{MgO}$ films under different sputtering condition. When the separation distance is between $5 \mathrm{~cm}$ and $6 \mathrm{~cm}$, the XRD peak of $\mathrm{MgO}$ (002) orientation appears at $\sim 19.4^{\circ}[12]$. However, the XRD peak of $\mathrm{MgO}$ film with separation distance $6 \mathrm{~cm}$ is weaker compared to those of separation distance 5 $\mathrm{cm}$. MgO films are almost amorphous at the separation distance ranging from 6 to $10 \mathrm{~cm}$. These results showed that the crystal status of $\mathrm{MgO}$ layer is sensitive to the separation distance. When the separation
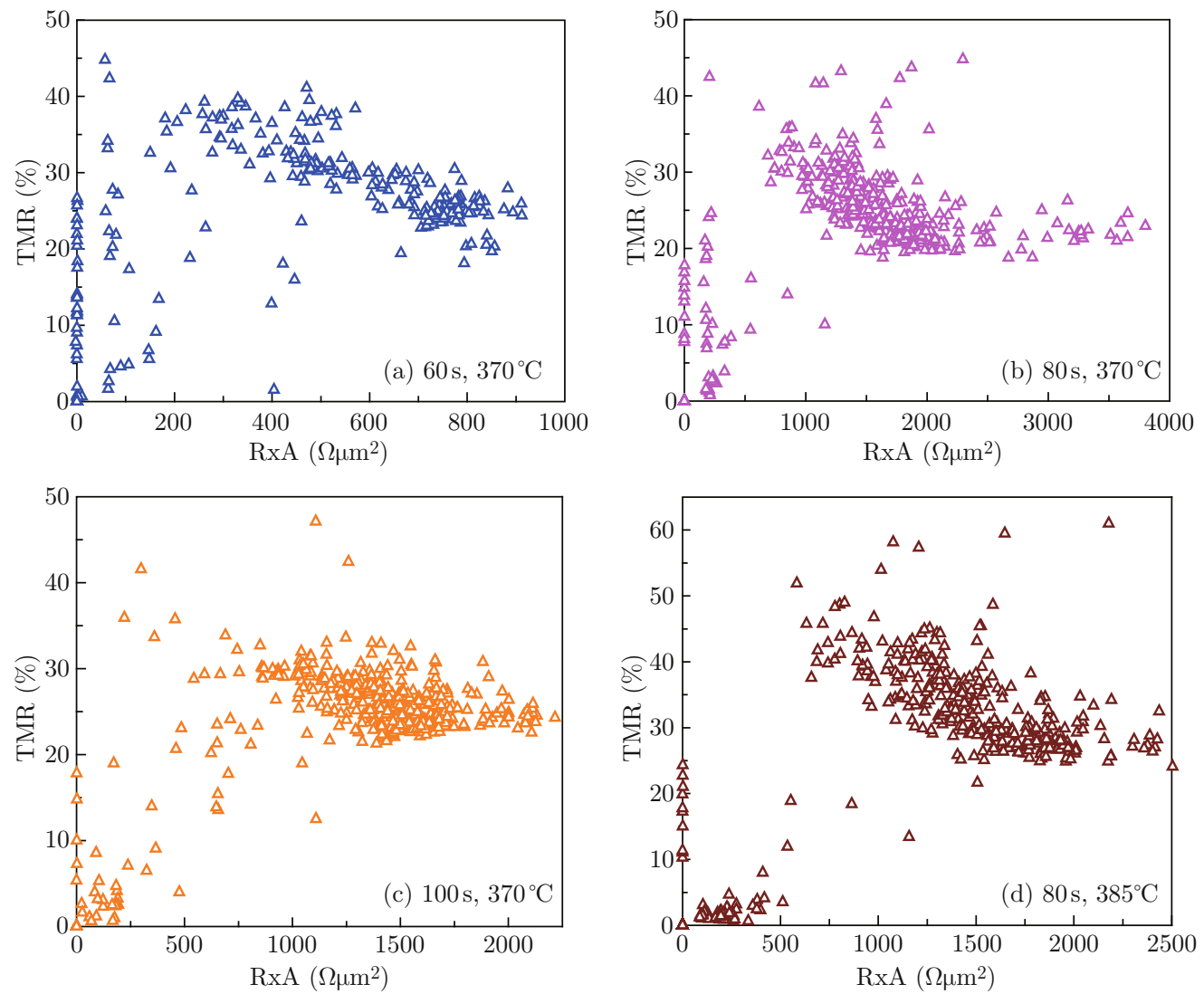

Fig. 2 TMR ratios versus RA products for MTJs with different oxidization time and annealing temperature. 


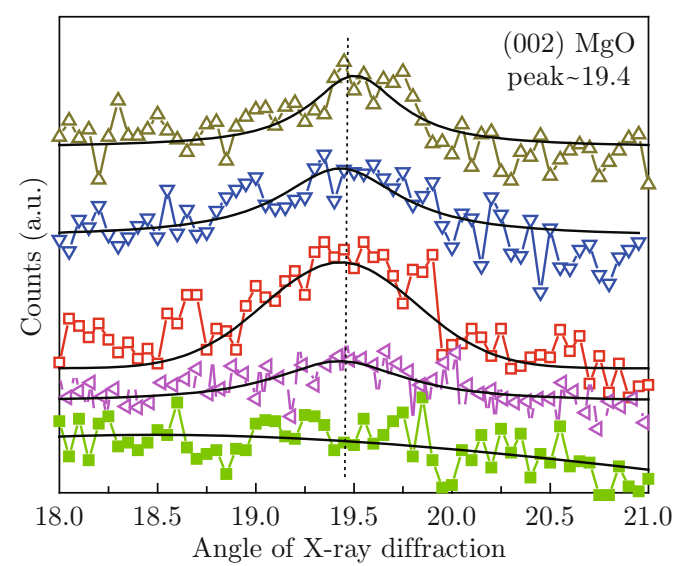

Fig. 3 X-ray diffraction scan curves of $\mathrm{MgO}$ films. Solid lines are fitting curves. The deposition conditions of $\mathrm{MgO}$ film: ( $\square$ ) RF power $170 \mathrm{~W}$, Ar pressure 12 mTorr, separation distance $5 \mathrm{~cm} ;(\mathbf{\square}) 170 \mathrm{~W}, 12 \mathrm{mTorr}$ and $9.5 \mathrm{~cm} ;(\nabla)$ $170 \mathrm{~W}, 5 \mathrm{~m}$ Torr and $5 \mathrm{~cm} ;(\triangle) 170 \mathrm{~W}, 8 \mathrm{~m}$ Torr and $5 \mathrm{~cm}$; $(\triangleleft) 170 \mathrm{~W}, 12 \mathrm{mTorr}$ and $6 \mathrm{~cm}$.

distance was set $5 \mathrm{~cm}$, the (002) $\mathrm{MgO}$ crystal structure was easily formed in the large range of RF power and Ar gas pressure.

Figure 4 shows the TMR curve of the representative MTJs. MgO barriers were deposited at the separation distance $5 \mathrm{~cm}$. The MTJs were annealed at $340^{\circ} \mathrm{C}$ for $1 \mathrm{~h}$. The MTJ MgO barrier thickness, RF sputtering power and Ar pressure were $19 \AA, 150 \mathrm{~W}$ and 12 mTorr (Fig. 4(a)), $15 \AA, 150 \mathrm{~W}$ and 12 mTorr (Fig. 4(b)), and $15 \AA, 100 \mathrm{~W}$ and 8 mTorr (Fig. 4(c)), respectively. The TMR ratios of MTJs are about $130 \%$ (Fig. 4(a)), 150\% (Fig. 4(b)) and 170\% (Fig. 4(c)), respectively. One sample with one kind of $\mathrm{MgO}$ barrier deposition condition can be fabricated $\sim 324$ MTJs. Here, we just chose a representative MTJ to plot a TMR curve under one deposition condition. From a statistical point of view, TMR ratios of most MTJs with above three kinds of $\mathrm{MgO}$ barrier deposition conditions are more than $100 \%$. Our results demonstrated that TMR ratios of most MTJs are always more than $100 \%$ for $\mathrm{MgO}$ barrier deposited with RF sputtering power ranging from $100 \mathrm{~W}$ to $200 \mathrm{~W}$, and Ar pressure ranging from $5 \mathrm{~m}$ Torr to $20 \mathrm{~m}$ Torr at the separation distance of $5 \mathrm{~cm}$. However, TMR ratios of MTJ are almost zero when the separation distance is larger than $6 \mathrm{~cm}$ due to amorphous $\mathrm{MgO}$ films. Therefore, the separation distance between $\mathrm{MgO}$ target and glass substrate is most important parameter to get high TMR ratio of $\mathrm{MgO}$ based MTJs in magnetic sputtering system Nordiko 2000.

\section{Conclusions}

In summary, $\mathrm{MgO}$ barriers were investigated by sputtering single crystal $\mathrm{MgO}$ target and natural oxidization, respectively. $\mathrm{Mg}$ layer thickness, oxidation time and oxygen flow rate are relative to TMR ratios under

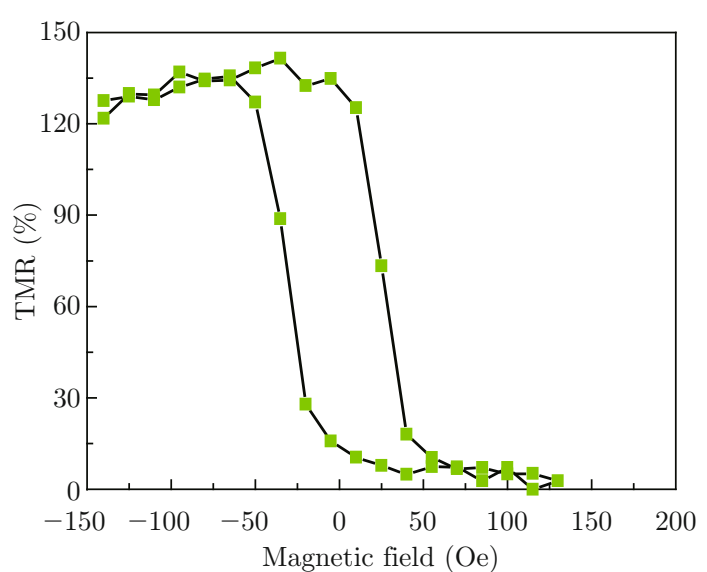

(a) RF $150 \mathrm{~W}, 8 \mathrm{~m}$ Torr, $\mathrm{MgO} 19 \AA$

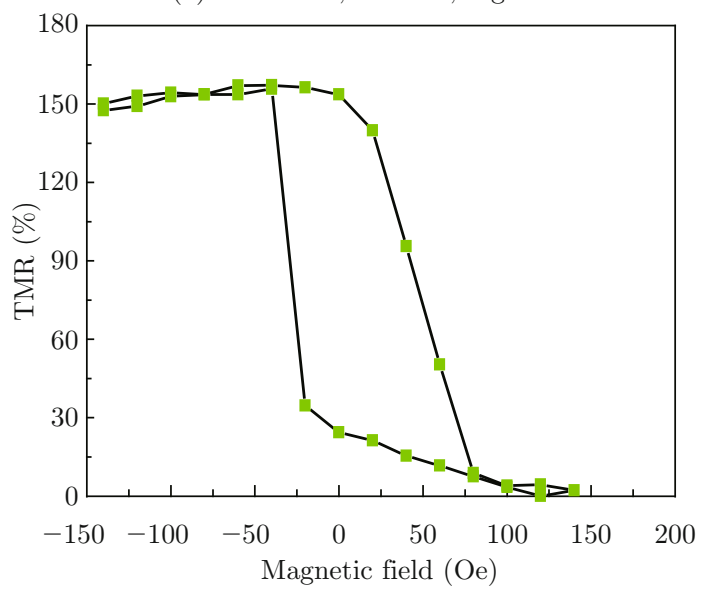

(b) RF $150 \mathrm{~W}, 12 \mathrm{mTorr}, \mathrm{MgO} 15 \AA$

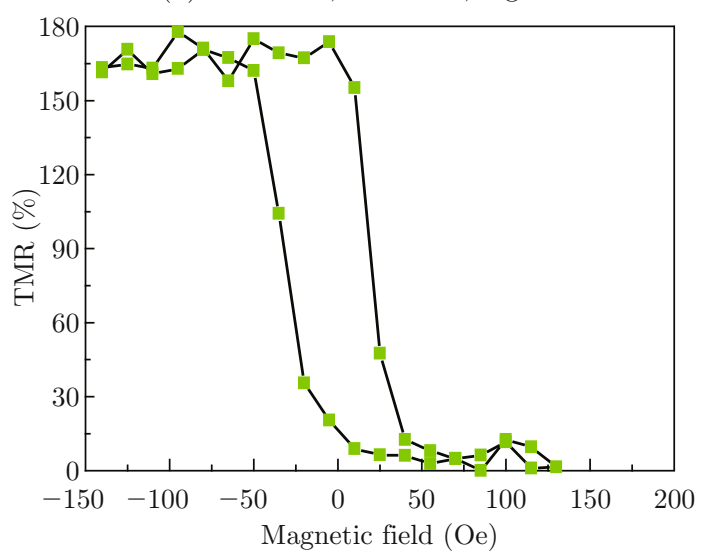

(c) RF $100 \mathrm{~W}, 12 \mathrm{~m}$ Torr, $\mathrm{MgO} 15 \AA$

Fig. 4 TMR curves of $\mathrm{MgO}$ barriers deposited with different deposition parameters.

natural oxidization method. The TMR ratio of the optimal MTJ is more than $60 \%$ at annealing temperature $385^{\circ} \mathrm{C}$. The separation distance of $\mathrm{MgO}$ target and substrate is more important for depositing (001) $\mathrm{MgO}$ crystal structure in magnetic sputtering system Nordiko 2000. The TMR ratios of reasonable MTJs are more than $100 \%$ at the separation distance of $5 \mathrm{~cm}$. The MgO film is almost amorphous when the separation distance varies from 6 to $10 \mathrm{~cm}$. 


\section{Acknowledgments}

Xiaohong Chen is grateful to the Natural Science Foundation of Shanghai Science and Technology Commission (grant No. 11ZR1411300) and Pujiang Talent Program of Shanghai Science and Technology Commission (grant No. 11PJ1402700) for the financial support.

\section{References}

[1] M. Julliere, Phys. Lett. A 54, 225 (1975). http://dx. doi .org/10.1016/0375-9601 (75) 90174-7

[2] S. Ikeda, J. Hayakawa, Y. Ashizawa et al., Appl. Phys. Lett. 93, 082508 (2008). http://dx.doi.org/ 10.1063/1.2976435

[3] H. X. Wei, Q. H. Qin, M. Ma et al., J. Appl. Phys. 101, 09B501 (2007).

[4] S. Maekawa and U. Gafvert, IEEE Trans. Magn. 18, 707 (1982). http://dx.doi.org/10.1109/TMAG. 1982. 1061834

[5] J. Nowak and J. Rauluszkiewicz, J. Magn. Magn. Mater. 109, 79 (1992). http://dx.doi.org/10.1016/ 0304-8853 (92) 91034-Q

[6] T. Miyazaki and N. Tezuka, J. Magn. Magn. Mater. 139, L231 (1995).
[7] W. H. Butler, X. G. Zhang, T. C. Schulthess et al., Phys. Rev. B 63, 054416 (2001). http://dx.doi.org/ 10.1103/PhysRevB.63.054416

[8] S. S. P. Parkin, C. Kaiser, A. Panchula et al., Nature Mater. 3, 862 (2004). http://dx.doi.org/10.1038/ nmat1256

[9] S. Yuasa, T. Nagahama, A. Fukushima et al., Nature Mater. 3, 868 (2004). http://dx.doi.org/10.1038/ nmat 1257

[10] W. G. Wang, C. Ni, A. Rumaiz et al., Appl. Phys. Lett. 92, 152501 (2008). http://dx.doi.org/10.1063/1. 2903147

[11] J. Cao, J. Kanak, T. Stobiecki et al., IEEE Trans. Magn. 45, 3464 (2009). http://dx.doi.org/10.1109/ TMAG. 2009. 2025382

[12] S. Cardoso, R. J. Macedo, R. Ferreira et al., J. Appl. Phys. 103, 07A905 (2008).

[13] S. Ikeda, J. Hayakawa, Y. M. Lee et al., Jpn. J. Appl. Phys. 44, L1442 (2005). http://dx.doi.org/10.1143/ JJAP . 44 . L1442

[14] J. Wang, P. P. Freitas, E. Snoeck et al., Appl. Phys. Lett. 79, 4387 (2001). http://dx.doi.org/10.1063/ 1.1421232

[15] R. Ferreira, P. P. Freitas, M. MacKenzie et al., Appl. Phys. Lett. 86, 192502 (2005). http://dx.doi.org/ 10.1063/1. 1925318 